\title{
PREPARAÇÃO DE AMOSTRA DE FÍGADO BOVINO E ESTUDO DE MICRO HOMOGENEIDADE PARA A DETERMINAÇÃO DE Cd E Pb POR ESPECTROMETRIA DE ABSORÇÃO ATÔMICA COM ATOMIZAÇÃO ELETROTÉRMICA E AMOSTRAGEM DIRETA DE SÓLIDO
}

\author{
Cassiana Seimi Nomura e Pedro Vitoriano Oliveira* \\ Instituto de Química, Universidade de São Paulo, CP 26077, 05513-970 São Paulo - SP
}

Recebido em 27/1/05; aceito em 3/8/05; publicado na web em 20/1/06

\begin{abstract}
BOVINE LIVER SAMPLE PREPARATION AND MICRO-HOMOGENEITY STUDY FOR Cd AND Pb DETERMINATION BY SOLID SAMPLING ELECTROTHERMAL ATOMIC ABSORPTION SPECTROMETRY. This work describes a systematic study for bovine liver sample preparation for $\mathrm{Cd}$ and $\mathrm{Pb}$ determination by solid sampling electrothermal atomic absorption spectrometry. Samples were prepared using different procedures: (1) drying in a household microwave oven followed by drying in a stove at $60{ }^{\circ} \mathrm{C}$ until constant mass, and (2) freeze-drying. Ball and cryogenic mills were used for grinding. Particle size, sample size and micro sample homogeneity were investigated. All prepared samples showed good homogeneity $\left(H_{e}<10\right)$ even for low sample mass, but samples dried in a microwave oven/stove and ground in a ball mill presented the best homogeneity.
\end{abstract}

Keywords: solid sampling atomic absorption; homogeneity; bovine liver

\section{INTRODUÇÃO}

A determinação de elementos em nível de traços em amostras biológicas como fluídos ${ }^{1,2}$, tecidos ${ }^{3}$ e órgãos ${ }^{4}$ é importante porque gera informações que auxiliam na resolução de problemas relacionados a estudos nutricionais, toxicológicos e bioquímicos. Em certos casos, a necessidade de informações sobre a ocorrência, distribuição e forma química de elementos nos materiais biológicos tem incentivado o desenvolvimento de procedimentos analíticos visando, além da determinação total, a especiação química ${ }^{5}$.

Uma das técnicas mais recomendadas para determinação de elementos traços em materiais biológicos é a espectrometria de absorção atômica com atomização eletrotérmica (ETAAS). As altas seletividade e sensibilidade, simplicidade nas operações e automação promovidas pelo amostrador automático, o que dispensa a atenção constante do analista, são características importantes que merecem destaque. Na maioria dos métodos, a preparação da amostra envolve apenas diluição em um solvente adequado e introdução direta no tubo de grafite.

A possibilidade de amostragem direta de sólidos (SS-ETAAS) ampliou o universo de aplicações da espectrometria de absorção atômica com atomização eletrotérmica, possibilitando a análise de pequenas massas de amostra, em intervalos que podem variar de 40 - $1000 \mu \mathrm{g}^{3,4,6-8}$, tornando-a muito conveniente quando a disponibilidade de amostra é limitada, por ex., em casos de biópsia de órgãos e tecidos ${ }^{3,4}$, em algumas investigações voltadas à ciência forense $\mathrm{e}^{7,8}$ e em estudos envolvendo a avaliação de homogeneidade de materiais ${ }^{9-12}$. No entanto, a obtenção de resultados precisos e exatos utilizando pequenas massas de amostras não é uma tarefa trivial, uma vez que massas inferiores a $100 \mathrm{mg}$ podem comprometer a representatividade, não somente pelos erros associados às pesagens como também pela falta de homogeneidade de certos materiais para alguns elementos ${ }^{13}$. Em geral, a variação dos resultados tende a piorar com a diminuição da massa amostrada.

*e-mail: pvolivei@iq.usp.br
Os materiais de referência certificados (MRC) disponíveis atualmente garantem a representatividade para massas de amostras acima de 100 ou $500 \mathrm{mg}^{9}$, as quais são muito superiores àquelas praticadas em SS-ETAAS. Porém, para alguns métodos de determinação elementar com amostragem direta de sólido, o uso de MRC é necessário para a calibração do equipamento e indispensável para testes de validação, mesmo não sendo recomendados para valores tão baixos de amostragem. Considerando essa limitação dos MRC para pequenas massas, a avaliação de procedimentos de preparação de amostras para determinações elementares em SS-ETAAS torna-se muito importante.

Nesse trabalho foram avaliados diferentes procedimentos de preparação de amostra de fígado bovino para determinação de $\mathrm{Cd}$ e $\mathrm{Pb}$ por SS-ETAAS. Os principais parâmetros estudados foram secagem, moagem, avaliação do tamanho de partículas, massa de amostra e sua relação com a homogeneidade. A homogeneidade das amostras foi avaliada para cada um dos procedimentos de secagem e moagem utilizados. A maneira de garantir a homogeneidade da amostra foi pelo uso de procedimentos de moagem eficientes.

\section{PARTE EXPERIMENTAL}

\section{Instrumentação}

Todas as determinações foram feitas em um espectrômetro de absorção atômica modelo ZEEnit ${ }^{\circledR} 60$ (AnalytikjenaAG, Jena, Alemanha) equipado com corretor de fundo transversal baseado no efeito Zeeman, atomizador recoberto com grafite pirolítico e aquecimento transversal, e plataforma de grafite pirolítico do tipo "boat". A transferência da plataforma da microbalança (Auto Balance AD-4, Perkin-Elmer, Norwalk, CT, USA) para o interior do tubo de grafite foi feita com um amostrador mecânico de sólidos (SSA-6 Z, AnalytikjenaAG). O espectrômetro foi operado com lâmpadas de catodo oco de $\mathrm{Cd}(\lambda=228,8 \mathrm{~nm}, \mathrm{i}=4,0$ $\mathrm{mA}$, resolução espectral $=0,8 \mathrm{~nm})$ e $\mathrm{Pb}(\lambda=217,0 \mathrm{~nm}, \mathrm{i}=4,0$ $\mathrm{mA}$, resolução espectral $=0,5 \mathrm{~nm})$. Todas as medidas foram baseadas nos valores de absorbância integrada. Argônio 99,998\% 
(v/v) (Air Liquide Brasil, São Paulo, Brasil) foi utilizado como gás protetor e de purga.

A secagem das amostras foi feita em forno de microondas doméstico (Cônsul - $300 \mathrm{~W}$ ), estufa (Fanen, $\left.300{ }^{\circ} \mathrm{C}\right)$, e liofilizador (E - C Micro Módulo).

As amostras foram moídas em moinho criogênico Spex 6750 Freezer Mill (Spex, Metychen, NJ, USA) e moinho de bolas 8000 M Spex CertPrep (Spex, Metuchen, NJ, USA), equipado com frascos e bolas de carbeto de tungstênio.

A medida do tamanho de partícula foi feita em analisador de partículas (Malvern Instruments, Malvern, UK) com unidade automatizada de dispersão de amostra MS-17.

Para as determinações de $\mathrm{Cd}$ e $\mathrm{Pb}$ em amostras digeridas foi utilizado o amostrador automático (AS 52, AnalytikJenaAG) do espectrômetro de absorção atômica com atomização eletrotérmica. As amostras foram previamente digeridas em forno de microondas com cavidade, modelo Multiwave 3000 (Anton Paar, Graz, Áustria).

\section{Reagentes e soluções}

Todas as soluções foram preparadas com água desionizada de alta pureza $(18 \mathrm{M} \Omega \mathrm{cm}$ ), obtida pelo sistema de ultrapurificação de água Milli-Q (Millipore, Bedford, MA, USA). Foram utilizados reagentes de grau analítico como o $\mathrm{H}_{2} \mathrm{O}_{2}$ (Merck, Darmstadt, Alemanha) e $\mathrm{HNO}_{3}$ (Synth, Diadema, SP, Brasil), sendo esse último purificado por destilação abaixo do ponto de ebulição em destiladores de quartzo (Marconi, Piracicaba, SP, Brasil). Soluções analíticas de referência de $\mathrm{Cd}$ (II) e $\mathrm{Pb}$ (II) em meio de $0,1 \% \mathrm{v} / \mathrm{v}$ de $\mathrm{HNO}_{3}$ foram preparadas após sucessivas diluições das soluções estoques de $1000 \mathrm{mg} \mathrm{L}^{-1}$ $\mathrm{CdCl}_{2}$ e $\mathrm{Pb}\left(\mathrm{NO}_{3}\right)_{2}$ (Titrisol, Merck).

Os modificadores químicos usados foram preparados por diluição de soluções contendo $1000 \mathrm{mg} \mathrm{L}^{-1} \mathrm{de} \mathrm{Na}_{2} \mathrm{WO}_{4} \cdot 2 \mathrm{H}_{2} \mathrm{O}$ (Merck), $\mathrm{RhCl}_{6}$ (Sigma, St. Louis, MO, USA), $\mathrm{NH}_{4} \mathrm{H}_{2} \mathrm{PO}_{4}$ (Merck), $\mathrm{Pd}\left(\mathrm{NO}_{3}\right)_{2}$ (Merck) e $\mathrm{Mg}\left(\mathrm{NO}_{3}\right)_{2}$ (Merck). Todas as soluções foram armazenadas em frascos de polipropileno descontaminados (Nalge Company, Rochester, NY, USA).

\section{Procedimento}

\section{Descontaminação dos materiais}

Toda a vidraria e frascos de polipropileno utilizados para preparo e armazenamento das amostras e soluções analíticas de referência foram lavados com detergente, enxaguados com água destilada, preenchidos com solução de ácido nítrico $10 \%$ v/v durante 24 h e, nova- mente, enxaguados com água destilada e desionizada. Após a descontaminação, os balões volumétricos e frascos para armazenamento das soluções analíticas de referência foram preenchidos com solução de ácido nítrico $10 \%$ v/v e somente antes da utilização esses materiais foram enxaguados com água bi-deionizada.

\section{Programa de aquecimento}

As determinações de $\mathrm{Cd}$ e $\mathrm{Pb}$ por ETAAS, após digestão da amostra, e por SS-ETAAS foram feitas seguindo os programas de aquecimento apresentados na Tabela 1. Esses programas foram otimizados (temperaturas e tempos) a partir do estudo de temperaturas de pirólise e atomização, executados na ausência e presença de modificadores químicos.

Para a determinação de $\mathrm{Cd}$ e $\mathrm{Pb}$ por ETAAS, com amostragem de solução, foram usados os modificadores químicos $5 \mu \mathrm{g} \mathrm{Pd}+3 \mu \mathrm{g} \mathrm{Mg}$ e $25 \mu \mathrm{g} \mathrm{NH} \mathrm{H}_{2} \mathrm{PO}_{4}$, respectivamente. A determinação direta de $\mathrm{Cd}$ por SS-ETAAS foi feita utilizando a mistura $250 \mu \mathrm{g} \mathrm{W}+200 \mu \mathrm{g}$ Rh como modificador químico permanente ${ }^{14}$. No caso do $\mathrm{Pb}$, a determinação direta foi possível na ausência de modificador químico.

As calibrações do espectrômetro, tanto para as determinações em soluções aquosas como com amostragem direta do sólido, foram feitas utilizando $10 \mu \mathrm{L}$ de solução contendo 1 a $8 \mu \mathrm{g} \mathrm{L}^{-1}$ de $\mathrm{Cd}$ e 2,5 a $20 \mu \mathrm{g} \mathrm{L}^{-1}$ de $\mathrm{Pb}$ em $0,1 \%$ v/v $\mathrm{HNO}_{3}$.

\section{Preparo da amostra}

Uma peça de fígado bovino $(\sim 5 \mathrm{~kg})$, adquirido em um açougue da cidade de São Paulo, foi lavada com água Milli-Q e a membrana protetora retirada com lâmina de aço inoxidável. Em seguida, o fígado foi quarteado seguindo procedimento indicado na literatura ${ }^{15}$.

Cerca de $1 / 4$ desse material foi liofilizado, durante $48 \mathrm{~h}$ e outro $1 / 4$ seco, inicialmente, em forno de microondas de uso doméstico (30 min, $10 \%$ de potência - $30 \mathrm{~W}$ ) seguido de secagem em estufa à $60{ }^{\circ} \mathrm{C}$ até peso constante (aproximadamente $48 \mathrm{~h}$ ). Tanto o material liofilizado como o seco em microondas/estufa foi moído em moinho de bolas com dois diferentes tempos de moagem (5 e $30 \mathrm{~min}$ ) e em moinho criogênico com um único tempo de moagem (6 min), seguindo três ciclos do programa de moagem: 5 min de pré-congelamento, 2 min de moagem e 1 min de congelamento.

Uma amostra de fígado bovino certificado (NIST 1577b) foi utilizada para validação dos métodos desenvolvidos e para avaliar a homogeneidade de pequenas massas de amostras.

Anteriormente às determinações, aproximadamente $200 \mathrm{mg}$ de amostra e do MRC foram digeridas em forno de microondas com cavidade, usando mistura oxidante diluída ${ }^{16}\left(1 \mathrm{~mL} \mathrm{HNO}_{3}+2 \mathrm{~mL}\right.$ $\mathrm{H}_{2} \mathrm{O}_{2}+3 \mathrm{~mL} \mathrm{H}_{2} \mathrm{O}$ ) e o seguinte programa de aquecimento (tempe-

Tabela 1. Programas de aquecimento para determinação de $\mathrm{Cd}$ e $\mathrm{Pb}$ em fígado bovino

\begin{tabular}{|c|c|c|c|c|}
\hline \multicolumn{5}{|c|}{ Programa de Aquecimento para SS-ETAAS } \\
\hline Etapa & Temperatura $\left({ }^{\circ} \mathrm{C}\right)$ & Rampa $\left({ }^{\circ} \mathrm{C} \mathrm{s}^{-1}\right)$ & Patamar (s) & $\begin{array}{c}\text { Vazão de Argônio } \\
\left(\mathrm{L} \mathrm{min}^{-1}\right)\end{array}$ \\
\hline Secagem & 130 & 10 & 10 & 1,0 \\
\hline Pirólise & $600^{a}, 700^{b}$ & 100 & 40 & 1,0 \\
\hline Atomização & $1700^{a}, 2000^{b}$ & $1700^{a}, 2000^{b}$ & $4^{a}, 3^{b}$ & 0 \\
\hline Limpeza & 2200 & 1200 & 3 & 1,0 \\
\hline \multicolumn{5}{|c|}{ Programa de Aquecimento para ETAAS } \\
\hline Secagem I & 100 & 10 & 10 & 1,0 \\
\hline Secagem II & 130 & 10 & 10 & 1,0 \\
\hline Pirólise & $500^{a}, 600^{b}$ & 100 & 15 & 1,0 \\
\hline Atomização & $1600^{a}, 1800^{b}$ & $1600^{a}, 1800^{b}$ & 4 & 0 \\
\hline Limpeza & 2200 & 1200 & 4 & 1,0 \\
\hline
\end{tabular}

${ }^{a} \mathrm{Cd},{ }^{b} \mathrm{~Pb}$ 
ratura $/{ }^{\circ} \mathrm{C}$; rampa/min; patamar/min): etapa $1: 140,5$, 1 ; etapa 2 : 180, 8, 2; etapa 3: 220, 4, 10.

\section{Estudo da homogeneidade da amostra}

$\mathrm{O}$ estudo da homogeneidade do material foi feito por meio da determinação de $\mathrm{Cd}$ e $\mathrm{Pb}$ em diferentes massas das amostras preparadas pelos procedimentos propostos e no material de referência certificado. Devido à dificuldade de pesar repetidas massas de amostras, a precisão e exatidão dos resultados foram avaliadas em diferentes intervalos de massas: 1 ( 1 a $20 \mu \mathrm{g}), 2$ (21 a $30 \mu \mathrm{g}), 3$ (31 a 40 $\mu \mathrm{g}), 4(41$ a $50 \mu \mathrm{g}), 5(51$ a $60 \mu \mathrm{g}), 6(61$ a $70 \mu \mathrm{g}), 7$ (71 a $80 \mu \mathrm{g})$, $8(81$ a $90 \mu \mathrm{g}), 9(91$ a $100 \mu \mathrm{g}), 10(101 \mathrm{a} 120 \mu \mathrm{g}), 11(121 \mathrm{a} 200 \mu \mathrm{g})$ para o Cd, e 1 (1 a $50 \mu \mathrm{g}), 2(51 \mathrm{a} 100 \mu \mathrm{g}), 3(101$ a $200 \mu \mathrm{g}), 4$ (201 a $400 \mu \mathrm{g}), 5(401$ a $600 \mu \mathrm{g}), 6(601$ a $800 \mu \mathrm{g}), 7(801$ a $1000 \mu \mathrm{g})$, $8(1001$ a $1200 \mu \mathrm{g}), 9(1201$ a $1400 \mu \mathrm{g})$ para o $\mathrm{Pb}$.

Para cada intervalo de massas foram feitas cinco determinações e os resultados foram avaliados quanto à exatidão e precisão. Os valores de concentração indicados nos gráficos (linha cheia) com os respectivos desvios padrão (linha tracejada) foram obtidos após as análises de $\mathrm{Cd}$ e $\mathrm{Pb}$ por ETAAS, com digestão das amostras.

\section{RESULTADOS}

\section{Preparo das amostras}

As primeiras etapas envolvidas no pré-tratamento de amostras, visando análise direta de sólidos por técnicas espectroscópicas, consistem na secagem e moagem do material. Em geral, os materiais com alto teor de água, como os tecidos biológicos, necessitam de uma etapa de secagem e moagem antes da determinação dos elementos de interesse. Considerando a análise direta de sólidos por SS-ETAAS, essas etapas tornam-se muito mais críticas devido à necessidade de garantir a homogeneidade, mesmo para massas diminutas de amostra, freqüentemente inferiores a $1000 \mu \mathrm{g}^{17,18}$.

Entre os dois procedimentos adotados para secagem, o uso de forno de microondas combinado com estufa parece ser mais susceptível às perdas de elementos por volatilização, apesar de estudos da literatura mostrarem que a volatilização de compostos também pode ocorrer durante a liofilização ${ }^{19}$. No entanto, perdas de $\mathrm{Cd}$ e $\mathrm{Pb}$ não foram observadas em nenhuma das amostras preparadas pelos dois procedimentos adotados de secagem e moagem.

A moagem das amostras foi feita utilizando moinhos de bolas e criogênico. Na moagem com moinho de bolas o processo de cominuição se dá devido ao impacto das bolas com a amostra. Geralmente, o atrito das bolas gera uma ligeira elevação da temperatura, que é maior com o tempo de moagem e que pode causar perdas dos analitos. Lima e colaboradore ${ }^{20}$ verificaram que o aquecimento gerado, mesmo durante longos tempos de moagem, não é suficiente para causar perdas de alguns elementos com características voláteis.

A moagem criogênica tem sido recomendada para amostras com alto teor de fibras e gorduras. O congelamento rápido gera falhas na estrutura cristalina dos materiais tornando-os mais quebradiços, facilitando a moagem ${ }^{21}$. Por ocorrer na temperatura de liquefação do nitrogênio $\left(-196^{\circ} \mathrm{C}\right)$ o risco de eventuais perdas de compostos por volatilização é inexistente.

O tamanho médio das partículas de fígado seco em forno de microondas combinado com estufa ou liofilizado e moído em moinho de bolas diminuiu com o aumento do tempo de moagem. Para os dois procedimentos de secagem considerados, a distribuição do tamanho de partículas mostrou uma redução em torno de 30\% no tamanho médio das partículas, quando o tempo de moagem variou de 5 para $30 \mathrm{~min}$. As amostras de fígado seco em forno de microondas combinado com estufa e moídas no moinho de bolas, durante 5 e $30 \mathrm{~min}$, apresentaram diâmetros médios de 130 e 90 m, respectivamente. Por outro lado, a amostra de fígado liofilizada e também moída no moinho de bolas, durante $5 \mathrm{~min}$, apresentou diâmetro médio de $90 \mu \mathrm{m}$.

As amostras de fígado moídas no moinho criogênico apresentaram diâmetros médios de partículas em torno de $64 \mu \mathrm{m}$, independente do tipo de secagem utilizado.

\section{Determinação de $\mathrm{Cd}$ e $\mathrm{Pb}$ em fígado por SS-ETAAS e ETAAS}

O programa de aquecimento para a determinação de $\mathrm{Cd}$ e $\mathrm{Pb}$ em SS-ETAAS foi otimizado com amostra de fígado certificado (NIST 1577b). Na ausência de modificador químico, as temperaturas de pirólise de $\mathrm{Cd}$ foram de $400{ }^{\circ} \mathrm{C}$ em solução aquosa e $600{ }^{\circ} \mathrm{C}$ com amostragem direta do sólido. O uso do modificador químico permanente $(250 \mu \mathrm{g} \mathrm{W}+200 \mathrm{Rh})$ foi indispensável porque aumentou a estabilidade térmica do $\mathrm{Cd}$ na solução aquosa para $600{ }^{\circ} \mathrm{C}$, enquanto que no sólido permaneceu $600{ }^{\circ} \mathrm{C}$. Esses dados indicam que o modificador permanente não foi efetivo na estabilização térmica das espécies de $\mathrm{Cd}$ presentes no fígado bovino. No entanto, a estabilização térmica do Cd em solução aquosa, igualando-se àquela obtida no sólido, permitiu a determinação direta utilizando calibração aquosa. Quando $400{ }^{\circ} \mathrm{C}$ foi usada na pirólise, o sinal de fundo gerado pela vaporização da matriz do sólido foi muito elevado, comprometendo o sinal analítico do Cd. Por esse motivo, o modificador químico permanente $250 \mu \mathrm{g} \mathrm{W}+200 \mu \mathrm{g}$ Rh foi adotado para a determinação direta de $\mathrm{Cd}$ nas amostras de fígado. $\mathrm{O}$ acréscimo de temperatura $\left(200{ }^{\circ} \mathrm{C}\right)$ foi indispensável para eliminação de parte dos concomitantes, minimizando possíveis interferências espectrais.

A temperatura de pirólise para o $\mathrm{Pb}$, na ausência de modificador químico, foi de $700{ }^{\circ} \mathrm{C}$ tanto em solução aquosa como para amostragem direta do sólido. Como observado para o $\mathrm{Cd}$, a ação do modificador químico permanente sobre o $\mathrm{Pb}$ na amostra de fígado bovino também não foi perceptível. No entanto, essa temperatura de pirólise foi alta o suficiente para eliminar parte dos concomitantes, evitando interferências espectrais. Nesse caso, a determinação direta de $\mathrm{Pb}$ foi feita com calibração aquosa e na ausência de modificador químico.

Os resultados da determinação de $\mathrm{Cd}$ e $\mathrm{Pb}$ no material de referência certificado de fígado bovino e nas amostras de fígado preparadas pelos diferentes procedimentos de secagem e moagem propostos estão apresentados nas Tabelas 2 e 3, respectivamente. As concentrações de $\mathrm{Cd}$ e $\mathrm{Pb}$ encontradas pelos métodos SS-ETAAS e ETAAS foram submetidas ao teste-t pareado e os resultados obtidos, quando comparados entre si e com os valores certificados, foram concordantes em um nível de confiança de 95\%. Resultados semelhantes, com o mesmo nível de confiança, foram obtidos para as amostras preparadas com os diferentes procedimentos de secagem e moagem (Tabela 3), indicando que não ocorreram perdas dos elementos durante as etapas de secagem e moagem.

As massas características, baseadas nas absorbâncias integradas e calculadas a partir das equações das retas, foram 1,2 pg para $\mathrm{Cd}$ e 7,3 pg para $\mathrm{Pb}$. Os limites de detecção foram calculados como sendo

Tabela 2. Resultados das determinações de $\mathrm{Cd}$ e $\mathrm{Pb}\left(\mathrm{mg} \mathrm{kg}^{-1}\right)$ em fígado bovino certificado por SS-ETAAS e ETAAS

\begin{tabular}{lccc}
\hline & $\begin{array}{c}\text { Material de Referência } \\
\text { Valor certificado } \\
\left(\mathrm{mg} \mathrm{kg}^{-1}\right)\end{array}$ & $\begin{array}{c}\text { SS-ETAAS } \\
\left(\mathrm{mg} \mathrm{kg}^{-1}\right)\end{array}$ & $\begin{array}{c}\text { ETAAS } \\
\left(\mathrm{mg} \mathrm{kg}^{-1}\right)\end{array}$ \\
\hline $\mathrm{Cd}$ & $0,50 \pm 0,03$ & $0,50 \pm 0,05$ & $0,45 \pm 0,08$ \\
$\mathrm{~Pb}$ & $0,129 \pm 0,003^{\mathrm{b}}$ & $0,147 \pm 0,009$ & $<$ L.D.* \\
\hline$*$ L. $\mathrm{D}_{\mathrm{Pb}}=0,09 \mu \mathrm{g} \mathrm{g}^{-1}$ & &
\end{tabular}


Tabela 3. Determinação de $\mathrm{Cd}$ e $\mathrm{Pb}$ nas amostras de fígado bovino após secagem em forno de microondas combinado com estufa e moagem em moinho de bolas (I) ou em moinho criogênico (II), e liofilização e moagem em moinho de bolas (III) ou em moinho criogênico (IV)

\begin{tabular}{|c|c|c|c|c|}
\hline \multirow[b]{2}{*}{ Pré-tratamento } & \multicolumn{2}{|c|}{$\mathrm{Cd}\left(\mathrm{mg} \mathrm{kg}{ }^{-1}\right)$} & \multicolumn{2}{|c|}{$\mathrm{Pb}\left(\mathrm{mg} \mathrm{kg}^{-1}\right)$} \\
\hline & SS-ETAAS ${ }^{*}$ & ETAAS & SS-ETAAS ${ }^{* *}$ & ETAAS \\
\hline I & $0,074 \pm 0,001$ & $0,075 \pm 0,002$ & $0,198 \pm 0,010$ & $0,194 \pm 0,011$ \\
\hline II & $0,076 \pm 0,006$ & $0,070 \pm 0,001$ & $0,220 \pm 0,028$ & $0,210 \pm 0,005$ \\
\hline III & $0,071 \pm 0,002$ & $0,070 \pm 0,002$ & $0,240 \pm 0,018$ & $0,222 \pm 0,001$ \\
\hline IV & $0,064 \pm 0,002$ & $0,074 \pm 0,002$ & $0,248 \pm 0,006$ & $0,218 \pm 0,006$ \\
\hline
\end{tabular}

*massa de amostra $>70 \mu \mathrm{g} ; * *$ massa de amostra $>800 \mu \mathrm{g}$

três vezes os desvios padrão de 20 medidas consecutivas da plataforma sem amostra, sendo que a mesma foi retirada e introduzida no tubo a cada repetição. Os valores obtidos foram $1,0 \mathrm{pg}$ para $\mathrm{Cd}$ e $15 \mathrm{pg}$ para $\mathrm{Pb}$. Os intervalos de massas de fígado considerados para a obtenção desses resultados foram de 40 a $120 \mu \mathrm{g}$ para a determinação de $\mathrm{Cd}$ e de 400 a $1400 \mu \mathrm{g}$ para a determinação de $\mathrm{Pb}$.

\section{Micro homogeneidade do material}

Nas determinações com amostragem direta de sólidos por SSETAAS é importante considerar a massa mínima representativa, que é a menor massa que pode ser utilizada de modo a obter resultados precisos e exatos. A massa mínima representativa (M) pode ser calculada utilizando a Equação proposta por Pauwels e colaboradores ${ }^{22}$ : $\mathrm{M}=\left(\mathrm{k}_{2}^{\prime} \cdot \mathrm{dp} / \mathrm{i}\right)^{2} \cdot \mathrm{m}$, na qual, $\mathrm{M}=$ massa mínima representativa, $\mathrm{k}_{2}^{\prime}=$ fator de distribuição, $\mathrm{dp}=$ desvio padrão relativo experimental, $\mathrm{i}=$ incerteza associada ao valor certificado, $\mathrm{m}=$ massa de amostra. Outro parâmetro considerado para a escolha da massa mínima foi o fator de homogeneidade $\left(H_{e}\right)$. $\mathrm{O}$ fator de homogeneidade, proposto por Kurfürst e colaboradores ${ }^{23}$, é uma maneira simples para caracterizar amostras sólidas quanto à homogeneidade. Está relacionado com a massa da amostra (mg) e o coeficiente de variação das medidas (RSD), o qual pode ser calculado segundo a Equação $H_{e}=(\mathrm{RSD}) \cdot \mathrm{m}^{1 / 2}$. Segundo os autores, materiais com $H_{e}<10$ são considerados homogêneos. Para cada procedimento de secagem e moagem foi feito um estudo sistemático para encontrar a massa mínima da amostra que respeitasse a precisão, exatidão e o fator de homogeneidade desejado $\left(H_{e}<10\right)$.

Os gráficos de concentração de $\mathrm{Cd}$ e $\mathrm{Pb}$ em função da massa amostrada, representada em intervalos de massa, estão apresentados nas Figuras 1 e 2. Em cada um dos casos, os valores das concentrações (linhas cheias) e desvios padrão (linhas pontilhadas) para $\mathrm{Cd}$ e $\mathrm{Pb}$ foram determinados por ETAAS, utilizando soluções
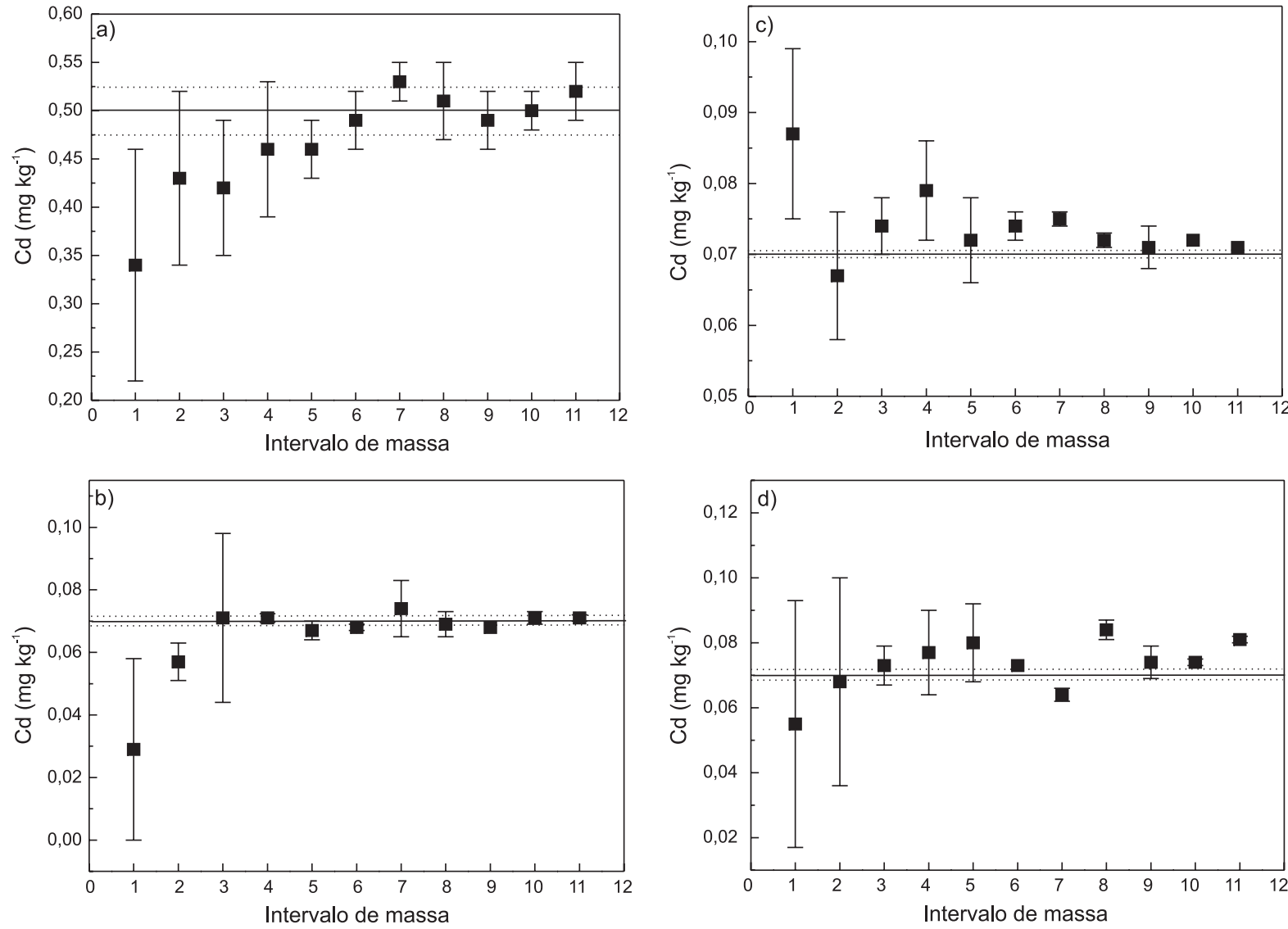

Figura 1. Estudo de homogeneidade para Cd em fígado bovino: (a) certificado (NIST 1577b); (b) seco em forno de microondas combinado com estufa e moído em moinho de bola por $30 \mathrm{~min}$; (c) liofilizado e moído em moinho de bola por 30 min e (d) seco em forno de microondas combinado com estufa e moído em moinho criogênico por $2 \mathrm{~min}$ 
aquosas e amostras digeridas em forno de microondas em frasco fechado: MRC (Tabela 2) e as amostras de fígado preparadas por diferentes procedimentos de secagem e moagem (Tabela 3). As determinações com soluções aquosas foram feitas com massas digeridas superiores a $250 \mathrm{mg}$ (massa mínima recomendada para o material de referência certificado de fígado NIST 1577b).

$\mathrm{Na}$ avaliação das figuras pode-se verificar que quanto menor a massa, pior a exatidão e a precisão dos resultados (Figuras 1 e 2), comprovando a afirmação previamente feita por Pauwels e colaboradores $^{22}$.

A determinação de Cd (Figura 1a) na amostra de fígado certificado apresentou $H_{e}<10$ mesmo quando massas em torno de $20 \mu \mathrm{g}$ foram amostradas, indicando boa homogeneidade do material. Calculando-se a massa mínima representativa conforme a Equação de Pauwels ${ }^{14}$, o valor encontrado foi de $1,17 \mathrm{mg}$. No entanto, na Figura 1a pode-se observar que resultados exatos, com valor de RSD $<8 \%$, podem ser obtidos utilizando-se massa mínima (70 $\mu \mathrm{g})$ muito menor que aquela encontrada teoricamente.

Os valores de $H_{e}$ encontrados para $\mathrm{Pb}$ no fígado certificado foram superiores a 10, mesmo para massas em torno de $1400 \mu \mathrm{g}$. Esses resultados indicam que o material não apresenta uma boa homogeneidade para $\mathrm{Pb}$, dificultando microanálises por SS-ETAAS. O certificado de análise desse material, fornecido pelo NIST, indica $250 \mathrm{mg}$ como a massa mínima de amostra que deve ser utilizada para garantir o valor certificado. Para a determinação do $\mathrm{Pb}, 11 \%$ foi o menor valor de RSD obtido para massa de $800 \mu \mathrm{g}$.

Os resultados das determinações de $\mathrm{Cd}$ nos diferentes intervalos de massas das amostras de fígado, seco em forno de microondas
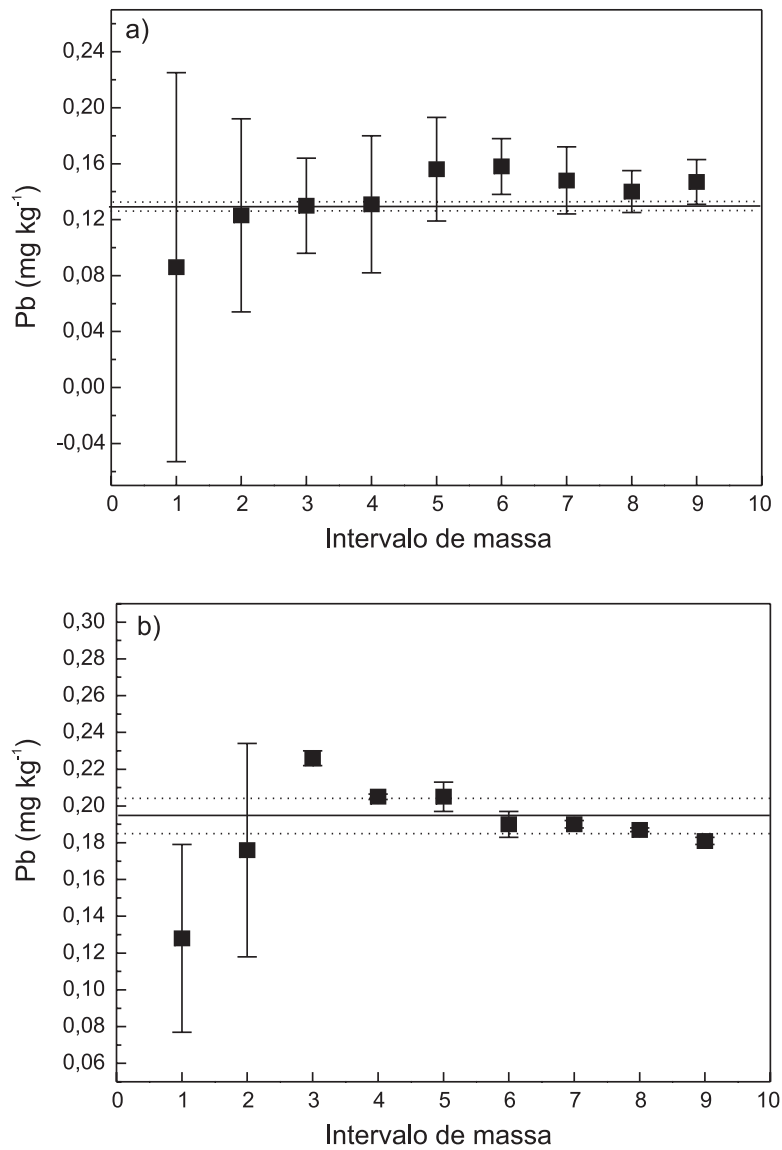

combinado com estufa e moídas em moinho de bolas (Figura 1b) ou criogênico (Figura 1d) seguiram uma mesma tendência. Em geral, foram observados desvios negativos nas concentrações de $\mathrm{Cd}$ para massas de amostra muito pequenas $(<40 \mu \mathrm{g})$. Porém, pode-se considerar o fígado seco em forno de microondas combinado com estufa e moído em moinho de bolas (Figura 1b) mais homogêneo. Nesse caso, os resultados concordaram em um nível de confiança de $95 \%$ (teste t-Student), para massas de amostras acima de $40 \mu \mathrm{g}$. Para o fígado liofilizado e moído em moinho de bolas (Figura 1c) foram observados elevados desvios padrão em cada intervalo. Esses desvios tornam-se menores e mais próximos do intervalo estabelecido quando massas de amostra superiores a $90 \mu \mathrm{g}$ são utilizadas. Apesar do fator de homogeneidade encontrado para $\mathrm{Cd}$ ter sido inferior a 10, para todos os intervalos de massa considerados, massas muito pequenas geraram resultados imprecisos e inexatos.

Em geral, as massas mínimas de fígado necessárias para determinação de $\mathrm{Pb}$ foram maiores que aquelas observadas para o $\mathrm{Cd}$. A menor sensibilidade do $\mathrm{Pb}$ em ETAAS, comparado com o Cd, é um dos fatores que deve ser considerado para justificar essas massas maiores. Comparando-se os resultados obtidos para os materiais preparados no laboratório, observa-se que o comportamento das amostras secas em forno de microondas combinado com estufa (Figuras 2b e 2d) também seguiu uma mesma tendência, independente do procedimento de moagem utilizado. Nessas amostras, os desvios padrão foram superiores a $25 \%$, quando massas de amostras inferiores a $400 \mu \mathrm{g}$ foram utilizadas. Para essas amostras, $H_{e}<10$ foram observados para massas superiores a $400 \mu \mathrm{g}$ (Figura $2 b$ e $2 d)$.
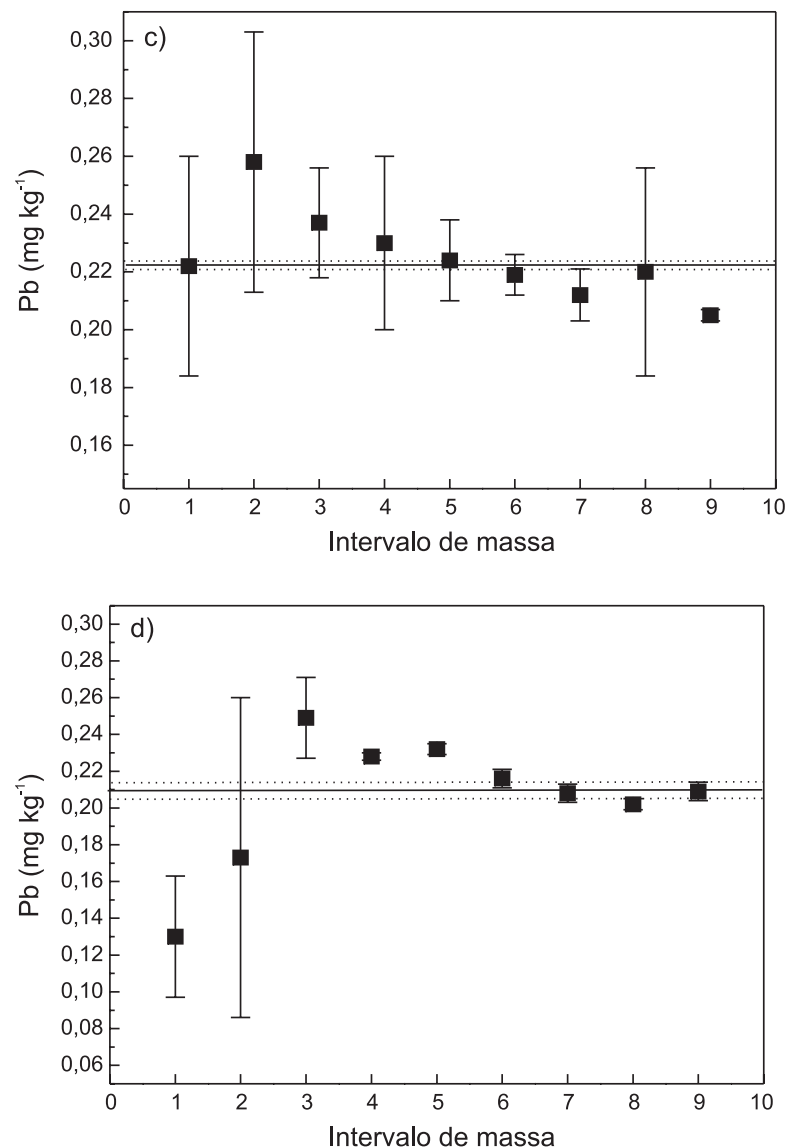

Figura 2. Estudo de homogeneidade para Pb em fígado bovino: (a) certificado (NIST 1577b); (b) seco em forno de microondas combinado com estufa e moído em moinho de bola por $30 \mathrm{~min}$; (c) liofilizado e moído em moinho de bola por $30 \mathrm{~min}$; e (d) seco em forno de microondas combinado com estufa e moído em moinho criogênico por 2 min 
$\mathrm{O}$ perfil do gráfico observado para $\mathrm{Pb}$ na amostra liofilizada (Figura 2c) foi muito semelhante àquele observado para o MRC (Figura 2a), cujo preparo também utiliza a liofilização para secagem. Nesse caso, resultados dentro do nível de confiança (95\%) foram obtidos somente quando massas superiores a $600 \mu \mathrm{g}$ foram analisadas.

\section{CONCLUSÃO}

As amostras de fígado moídas no moinho criogênico apresentaram diâmetros médios de partículas em torno de $64 \mu \mathrm{m}$, independente do tipo de secagem utilizado, indicando que o congelamento, seja durante o processo de secagem (liofilização) ou durante o processo de moagem (criogenia), facilita a moagem, proporcionando menores tamanhos de partículas.

As amostras de fígado bovino, preparadas pelos diferentes procedimentos de secagem e moagem, apresentaram boa homogeneidade para $\mathrm{Cd} \mathrm{e} \mathrm{Pb}\left(H_{e}<10\right)$, mesmo para massas inferiores a $1000 \mu \mathrm{g}$, como aquelas praticadas nas microanálises por SS-ETAAS. O estudo de micro homogeneidade mostrou que todos os procedimentos utilizados geraram resultados precisos e exatos para $\mathrm{Cd} \mathrm{e} \mathrm{Pb}$. Porém, com fígado seco em forno de microondas combinado com estufa e moído em moinho de bolas (30 min) foram possíveis amostragens de $40 \mu \mathrm{g}$ de amostra para determinação de $\mathrm{Cd}$ e $400 \mu \mathrm{g}$ para determinação de $\mathrm{Pb}$.

Embora o material certificado (NIST 1577b) tenha a homogeneidade garantida somente para massas superiores a $250 \mathrm{mg}$, foi possível verificar que massas de, aproximadamente, $60 \mu \mathrm{g}$ podem ser utilizadas para determinação do $\mathrm{Cd}$. No entanto, devido às baixas concentrações do $\mathrm{Pb}$ no fígado certificado, massas inferiores a $1400 \mu \mathrm{g}$ não apresentaram resultados satisfatórios em termos de precisão e exatidão.

Os resultados obtidos com os métodos de preparação de amostra utilizados no trabalho indicaram a possibilidade de produzir fígado bovino de referência para determinação de $\mathrm{Cd}$ e $\mathrm{Pb}$ por SS-ETAAS.

\section{AGRADECIMENTOS}

À FAPESP e ao CNPq pelo suporte financeiro. Os agradecimentos ainda se estendem ao Prof. F. J. Krug (CENA/USP), ao Prof. J. A.
Nóbrega do (DQ/UFSCar) e à Dra. A. R. A. Nogueira (EMBRAPA/ São Carlos) pelo empréstimo dos moinhos criogênico, de bolas e liofilizador. C. S. Nomura agradece à FAPESP e P. V. Oliveira, ao CNPq, pelas bolsas concedidas.

\section{REFERÊNCIAS}

1. Correia, P. R. M.; Nomura, C. S.; Oliveira, P. V.; Anal. Sci. 2003, 19, 1519.

2. Correia, P. R. M.; Oliveira, P. V.; At. Spectrosc. 2004, 24, 222.

3. Herber, R. F. M.; Roelofsen, A. M.; Roelfzema, W. H.; Peere-Stegeman, J. H. J. C.; Fresenius Z. Anal. Chem. 1985, 322, 743.

4. Nordahl, K.; Radziuk, B.; Thomassem, Y.; Weberg, R.; Fresenius J. Anal. Chem. 1990, 337, 310 .

5. De La Guardia, M.; Cervera, M. L.; Morales-Rubio, A. I.; Speciation Studies by Atomic Spectroscopy, JAI Press Inc.: New York, 1999.

6. Jackson, K. W.; Electrothermal Atomization for Analytical Atomic Spectrometry, John Willey \& sons: New York, 1999.

7. Hahn, E.; Hahn, K.; Mohn, C.; Stoeppler, M.; Fresenius J. Anal. Chem. 1990, 337, 306.

8. Lichtenberg, W.; Fresenius Z. Anal. Chem. 1987, 328, 367.

9. Rossbach, M.; Ostapczuk, P.; Emons, H.; Fresenius J. Anal. Chem. 1998, $360,380$.

10. Pauwels, J.; Kurfürst, U.; Grobecker, K. H.; Quevauviller, P.; Fresenius J. Anal. Chem. 1993, 345, 478.

11. Bagschik, U.; Quack, D.; Stoeppler, M.; Fresenius J. Anal. Chem. 1990, $338,386$.

12. Mohl, C.; Grobecker, K. H.; Stoeppler, M.; Fresenius Z. Anal. Chem. 1987, $328,413$.

13. Pawels, J.; Kramer, G. N.; De Angelis, L.; Grobecher, K. H.; Fresenius J. Anal. Chem. 1990, 338, 515.

14. Nomura, C. S.; Correia, P. R. M.; Oliveira, P. V.; Oliveira, E.; J. Braz. Chem. Soc. 2004, 15, 75.

15. Lücker, E.; König, H.; Gabriel, W.; Rosopulo, A.; Fresenius J. Anal. Chem. 1992, 342, 941.

16. Araújo, G. C. L.; Gonzalez, M. H.; Ferreira, A. G.; Nogueira, A. R.; Nóbrega, J. A.; Spectrochim. Acta, Part B 2002, 57, 2121.

17. Nakamura, T.; Oka, H.; Morikawa, H.; Sato, J.; Analyst 1995, 120, 131.

18. Iyengar, G. V.; Kasperek, K.; J. Radioanal. Nucl. Chem. 1977, 39, 301.

19. Uchino, E.; Jin, K.; Tsuzuki, T.; Inue, K.; Analyst 1987, 112, 291.

20. Lima, E. C.; Krug, F. J.; Ferreira, A. T.; Barbosa Junior, F.; J. Anal. At. Spectrom. 1999, 14, 269

21. Koglin, D.; Backhaus, F.; Schaladot, J. D.; Chemosphere 1997, 34, 2041.

22. Pawels, J.; Hofmann, C.; Vandecasteele, C.; Fresenius J. Anal. Chem. 1994, $348,418$.

23. Kurfürst, U.; Fresenius Z. Anal. Chem. 1983, 315, 304. 